Original Research

\title{
Naming and Framing the Behavioural and Psychological Symptoms of Dementia (BPSD) Paradigm: Professional Stakeholder Perspectives
}

\author{
Emma Wolverson ${ }^{1,}{ }^{*}$, Heather Birtles ${ }^{2}$, Esme Moniz-Cook ${ }^{1,}{ }^{*}$, Ian James ${ }^{2}$, Dawn Brooker ${ }^{3}$, Frances \\ Duffy $^{4}$
}

1. Faculty of Health Sciences, University of Hull, Hull, UK; E-Mails: e.wolverson@hull.ac.uk; e.d.moniz-cook@hull.ac.uk

2. Northumberland, Tyne and Wear NHS Foundation Trust, Newcastle upon Tyne, UK; E-Mails: H.Birtles@newcastle.ac.uk; lanAndrew.James@ntw.nhs.uk

3. Association for Dementia Studies, University of Worcester, Worcester, UK; E-Mail: d.brooker@worc.ac.uk

4. Northern Health and Social Care Trust, Northern Ireland, UK; E-Mail: Frances.Duffy@northerntrust.hscni.net

* Correspondences: Emma Wolverson, Esme Moniz-Cook; E-Mails: e.wolverson@hull.ac.uk; e.d.moniz-cook@hull.ac.uk

Academic Editor: James S. Powers

OBM Geriatrics

2019, volume 3 , issue 4

doi:10.21926/obm.geriatr.1904080
Received: September 08, 2019

Accepted: October 09, 2019

Published: October 11, 2019

\begin{abstract}
Background: Behavioural and Psychological Symptoms of Dementia and Challenging Behaviour in dementia are just two of a variety of terms for a complex paradigm that covers the most distressing and costly aspects of the condition. The terminology used to describe these aspects can influence what is measured as outcomes and what is considered as evidence of improvement. Unhelpful or outmoded narratives could be a barrier to developing innovative interventions or in determining what works for whom. This UK study explored professional opinions about commonly used terminology in this paradigm.
\end{abstract}

Methods: This mixed methods study involved wide-ranging multidisciplinary professionals and stakeholders. A consultation event was attended by 74 multi-disciplinary professionals. Written feedback from this event was used to develop an online survey. The survey was

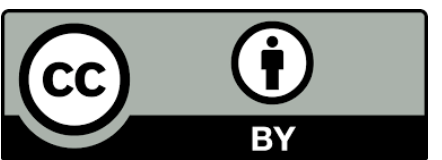

(C) 2019 by the author. This is an open access article distributed under the conditions of the Creative Commons by Attribution License, which permits unrestricted use, distribution, and reproduction in any medium or format, provided the original work is correctly cited. 
disseminated using a cascading snowballing method through multi-professional groups. Survey respondents ranked preferences for terms and stated reasons for their choices. Thematic content analysis was used to explore patterns of meaning.

Results: From the consultation event a list of 14 common terms were generated and formed the basis of the on-line survey. 378 respondents completed the survey. There was a wide variation across professional groups on preferred terminology with 'unmet need', 'behaviour that challenges', 'BPSD' and 'stress and distress' being ranked as the first choice by the majority. Five themes emerged from the qualitative data, revealing important nuances and challenges in relation to terminology.

Conclusions: Words have the power to shape thoughts, beliefs, emotions and behaviour. In line with the international advocacy movement, our UK findings suggest that future international consensus should, in addition to multi-professional and stakeholder experts, involve wide-ranging groups of people with dementia, their families and advocates. This would ensure that we use descriptive language, that does not objectify peoples' experience and that can be easily understood by all.

\section{Keywords}

Dementia; behaviour; BPSD; distress; psychological symptoms; professional survey

\section{Introduction}

Behaviour changes in dementia were a neglected research area until the mid-1980's [1] when studies appeared investigating the nature of behavioural disturbance (see [2]). In 1996, an international consensus group reframed behavioural disturbances as 'Behavioural and Psychological Symptoms of Dementia (BPSD) defined as 'signs and symptoms of disturbed perception, thought content, mood or behaviour that frequently occur in patients with dementia' $[3,4]$. BPSD has also been described as Neuropsychiatric Symptoms (NPS) [5]. The original consensus group was sponsored by the pharmaceutical industry [6] and was followed by texts on the use of acetylcholinesterase inhibitors showing improvements in behavioural symptoms ([7] pp 562) and clinical guidance on antipsychotic treatment ([7] pp 572 Table 14.5). More recently, Chen and colleagues [8] note that acetylcholinesterase inhibitors may not have demonstrated a significant preventative impact on BPSD. In the UK, concern about use of antipsychotic agents in dementia, resulted in policy-driven moves to change practices in England [9, 10], followed by National Health Service (NHS) targets for the reduction of antipsychotics and recommendations against these for first-line management [11].

\subsection{Historical UK Perspectives}

As far back as 1995, the Social Psychologist Tom Kitwood [12] had reconceptualised 'problem behaviours' in dementia as part of an 'old culture' of care. In the new culture of person-centred care, Kitwood proposed that all behaviours should primarily be seen as a communication of unmet need ([12] p10) expounding these theories more fully in his later book ([13] pp 82-85, [14]). 
Kitwood's work on person-centred care forms the basis of the National Institute for Health and Care Excellence (NICE) Guidelines that emphasises the importance of a supportive social and psychological environment to minimise the occurrence of such behaviours due to poor quality care. In 2000, the Clinical Psychologist Graham Stokes [15] was one of the first to introduce the term 'Challenging Behaviour' (CB) in dementia, thus shifting the paradigm from a predominantly symptomatic approach towards person-centred perspectives for understanding the causes for behaviour. The term has since been used in the psychosocial literature for this paradigm, [16-21] defined as 'a manifestation of distress or suffering for the person with dementia, or of distress in the carer' [17]. A subtle change in name to 'Behaviours that Challenge' (BtC), meaning behaviours that challenge 'others' appeared in 2006, in England's Dementia NICE guidelines [22]. This incorporated clinical and social constructs, shifting the focus away from the individual with dementia, towards charging professionals and services to find solutions. BtC has been defined as 'actions that detract from the wellbeing of individuals due to the physical or psychological distress they cause within the settings that are performed'([23] p12). Its core construct is that 'unmet need' contributes to actions ('behaviours') which are an attempt by a person with dementia to enhance or maintain wellbeing or to ease their distress [24]. However BtC has not been widely used outside the UK; and the updated 2018 NICE guidance in England replaced the term BtC with 'Non-Cognitive Symptoms'(see recommendation 1.7; [25]). Elsewhere, an Australian government initiative in 2012, emphasised the caregiving context in its 'Reducing Behaviours of Concern (ReBOC)' guide [26], but the 2016 Australian clinical guidelines for dementia retained the term BPSD (see recommendations 77-98 pp 15-18 [27]). Despite efforts in some countries to reframe the BPSD paradigm towards concepts that reflect the multiple causes of behaviour in dementia care, the psychiatric term BPSD appears to continue to dominate the literature.

\subsection{Terminology: Updating the Concept}

Irrespective of its psychiatric or psychosocial perspective, the paradigm often outlines endpoint symptoms such as agitation, depression, apathy, delusions, hallucinations, aggression, wandering, restlessness, sexual disinhibition, anxiety, irritability, euphoria and sleep disturbances. The BPSD consensus of the late 1990s, outlined the two groups of 'psychological (meaning psychiatric) and behavioural (meaning observable) symptoms' [4]. Elaborating on this, Ballard and colleagues [28] suggested four sub-categories of agitation, psychosis, mood disorders and other. The inherent difficulties in categorisation BPSD are evident in the authors' classification where both 'agitation' and 'mood disorder' include anxiety; aggression is described as a consequence of agitation (and therefore by implication the same construct) [29]. Problems occur because the terms are conflated semantically and there is no theoretical or empirical evidence that one consistently mediates the other (ie. agitation always leads to aggression) $[17,30]$. This mixing of BPSD symptoms is also seen in bio-medical and pharmaceutical studies that combine concepts of agitation and aggression [31-33] when their neurobiology may be distinct [34]. A later international group consensus focussed on their original construct of agitation, introducing the term 'distress' but retained its definitional scope to 'excessive motor activity' and aggression in people with dementia $[35,36]$.

Additional names for this paradigm are emerging, such as 'distress' and 'stress and distress' which has gained widespread traction in Scotland [37]. England's 2018 Dementia NICE Guideline 
described 'non cognitive symptoms' as 'distress (in the person with dementia), agitation, aggression and psychosis' (see 1.7 [25]). Recently people with dementia and their advocates have stimulated, through social media (see for example [38]) an important and powerful debate about this topic. A number of people living with dementia have described terms as pejorative and outdated.

\subsection{Terminology: Outcome Measurement and Practice}

These shifting approaches to the paradigm have implications for outcome measurement and how research is translated into tools to guide practice. For example, England's NICE 2019 Quality Standard for this paradigm Managing Distress ([39] Quality Statement 6 pp 26) limits measurement of 'success' to reduction in antipsychotic prescribing rates ([39] pp 27). Proximal outcomes such as reduction in distress or reported behavioural symptoms are not seen as relevant targets for this paradigm. Measures of improvement in caregiver efficacy, coping, or distress are also absent. Poor conceptual clarity in this paradigm can hamper trustworthy outcomes from high quality applied research studies and their translation into evidence-based practice. This in turn can undermine communication to find constructive solutions for some of the challenges of living with dementia for people and those that offer them support.

\subsection{Rationale}

In 2018, together with a Call to Action aimed at commissioners of services [24], a programme to produce a set of best practice guidelines on how to deliver interventions for BtC was funded in the UK by the British Psychological Society (BPS). England's updated Dementia NICE guidance also appeared at this time [40]. One of several steps within this programme of work, aimed to examine understandings of terminology for this complex paradigm. The present study draws on knowledge and views of wide-ranging professionals from a variety of disciplines across the UK. This paper aims to detail the differing views of professionals and stakeholders on terminology and explore with them why and in what way does terminology for this paradigm matter?

\section{Methods}

\subsection{Data Collection}

The views of professionals working in the field of dementia care in the UK were explored. This paper presents the data from two consecutive phases:

Phase One: a one-day consultation event organised by the British Psychological Society to review the guidelines and examine practice associated with Behaviours that Challenge $(\mathrm{BtC})$ in dementia care. This was attended by 74 professionals from psychology (50\%), nursing (14\%), psychiatry (8\%), occupational therapy (8\%), and other professions e.g. General Practitioner, pharmacy, physiotherapy; care practitioners, social work, family carers, and those employed by dementia charities ( $n=1-3$ participants each). Participants were recruited via email from a network of clinical psychologists who worked in services that provided care for people with dementia and from key professional organisations (e.g. Royal Colleges of Psychiatry, GP, Nursing and Occupational Therapy), third sector representation and family carers. The issue of language was 
part of the consultation and participants were invited to provide written responses regarding terminology. Fifty-six participants provided written responses. From this, a list of 14 commonly used terms were generated.

Phase Two: These 14 terms were included in an online survey using Qualtrics online survey software. This was shared with people who attended the workshop and wider stakeholders including additional professionals to those of phase one, who had a specific interest in the topic, for example from medicine (Geriatricians/ GPs/ Neurology), nursing, speech therapy, social work and experts who had published in peer review journals. A cascading snowballing method for recruitment was used. Participants' could add to 14 terms, using the option 'other'. They ranked their preferred terms with reasons for their choice(s) and then described the advantages of their most preferred term. Finally, they indicated terms that they strongly disliked and stated their reason(s) for this. The survey was open for six weeks (May/June 2019) and was completed by 378 participants.

The project was approved by the University of Hull Faculty of Health Sciences Ethics committee.

\subsection{Data Analysis}

Qualtrics survey software was used with a bespoke spreadsheet for data extraction of quantitative data. This included preference and descriptive statistics for responses. Thematic analysis was used to identify and explore patterns of meaning of participants' expectations about a term and why language might be important in this area. Thematic analysis was selected as it is best suited to explaining the conceptualisation that a group holds on a particular topic [41]. This method of analysis can highlight similarities and differences across participants. This was important because of our interest in comparing the opinions of stakeholders about terminology. In thematic analysis, analysts hold a realist position; they believe that the data represents the meanings that participants have assigned to their experiences and convey their reality [42]. An inductive approach was used where data were coded without a pre-existing coding frame. Themes were identified as those aspects of the data that captured something important in relation to the overall research question.

Data was analysed using Braun and Clarke's [42] approach to thematic analysis, which involves of six phases of coding and theme development. Two researchers read and re-read the data, making a note of any initial analytic observations. They then engaged in a process of systematic data coding, identifying key features of the data initially in relation to each term, before examining for broader patterns of meaning across the whole data set. After a process of review and refinement which all authors of this paper contributed to, five themes were generated from the data. Writing this paper constituted the final phase of analysis where illustrative quotes were selected to bring together a coherent narrative.

\section{Results}

\subsection{Quantitative Results}

Table 1 shows the occupation of participants who completed the online survey in Phase Two. The majority (40\%) were Nurses, but most professional groups and supporting staff contributed, and some family carers/third sector stakeholders also completed questions on terminology. 
Table 1 Online survey - participants' occupations.

\begin{tabular}{|c|c|}
\hline & No. of Participants \\
\hline Occupation & $\begin{array}{l}n=378 \quad(\%-\text { rounded } \\
\text { up) }\end{array}$ \\
\hline $\begin{array}{l}\text { Nurse: Nurse unspecified }(n=93) \text {; Admiral Nurse }(n=51) \text {; Community } \\
\text { Psychiatric Nurse }(n=3) \text {; Assistant Practitioner }(n=3) \text {; Clinical Lecturer } \\
(n=2)\end{array}$ & $152(40 \%)$ \\
\hline Clinical Psychologist: Clinical ( $n=83)$; Assistant $(n=10) ;$ Trainee $(n=4)$ & $97(26 \%)$ \\
\hline $\begin{array}{l}\text { Medical Professions: Psychiatrist }(n=38) ; \text { GPs }(n=10) \text {; Geriatrician }(n=1) \\
\text { / Neurologist }(n=1)\end{array}$ & $50(13 \%)$ \\
\hline $\begin{array}{l}\text { Allied Health Professions/Pharmacy/Social Work: Occupational } \\
\text { Therapist }(n=24) \text {; Social Worker }(n=8) ; \text { Physiotherapist }(n=1) \text {; Speech \& } \\
\text { Language Therapist }(n=2) ; \text { Pharmacist }(n=1)\end{array}$ & $36(10 \%)$ \\
\hline Other: & $43(11 \%)$ \\
\hline $\begin{array}{l}\text { Support Workers/Dementia Advisors }(n=14) \text {; Hands-on care staff }(n=6) \text {; } \\
\text { Family carer }(n=5) \text {; Person with Dementia }(n=1) \text {; Dementia Researcher } \\
(n=7) \text {; Dementia Commissioning Manager/Service Lead/Ward } \\
\text { Administrator }(n=3) \text {; Unspecified }(n=7)\end{array}$ & \\
\hline
\end{tabular}

All 378 participants completed the rankings. Table 2 illustrates the average rankings for the 14 terms that had been generated in phase 1 . 'Unmet need' was most frequently ranked as $1^{\text {st }}$ choice, with $25 \%$ of participants choosing this as their most preferred. Unusual behaviours and socially unacceptable behaviours were never selected as 'most preferred' terms. "Other" was left blank in 95\% of responses suggesting the list captured most people's preferred term. Twenty-one participants commented on option 15 ("Other") but many of these terms were derivatives of the 14 terms listed. For example: 'Distressed Responses' / 'Behaviour and Situations that Challenge' / Behaviour/understandable responses / 'Communications of Need' / 'Dementia-related symptoms'. However the family context was also noted within this section - for example one person suggested the term 'Manipulative Behaviours or Attention-seeking Behaviours', whilst others noted 'Difficult for families' / 'My wife is not always distressed but I am - does this count?'.

Taking the 'average' (mean) score, "Stress and Distress" appeared to be the most "generally preferred' term, but large standard deviations suggest high variability among professional groups and stakeholders. Although there was not enough data for fine-grained analysis of terms by professional discipline, "Stress and Distress" was most preferred for Nurses (13\%) and Clinical Psychologists (29\%). However "BtC" was the most preferred terms for Occupational Therapists (25\%) and Psychiatrists (21\%). GPs preferred "BPSD" (38\%); and for the small group of family carers/one person with dementia, 50\% preferred "Unmet Need".

To better understand participants' preferences, the frequency with which each term was placed in the top five was reviewed (see Figure 1; the red line reflects $50 \%(n=189)$ of participant 
responses. "Stress and Distress" appeared most frequently with $73 \%$ of people ranking it in their top five, in contrast to "Socially Unacceptable Behaviour" which was only placed in the top five by 6 participants. Assuming that the top five rankings are salient features of terminology for this paradigm, only five terms appear important for the majority of participants; BtC, Stress and Distress, Distress, Distressed Behaviours and Unmet Need, and of these three reflect aspects of the term 'distress'. If terms associated with distress are amalgamated, the term BPSD also appears important for some.

Table 2 Ranking of terms.

\begin{tabular}{llll}
\hline Term & Mean & SD & Times ranked No.1 \\
\hline Unmet Need & 4.82 & 3.91 & 96 \\
Behaviours that Challenge (BtC) & 4.75 & 3.41 & 74 \\
BPSD & 6.85 & 4.20 & 67 \\
Stress and Distress & 4.33 & 2.52 & 53 \\
Distress & 4.79 & 2.47 & 25 \\
Distressed Behaviour & 4.84 & 2.43 & 21 \\
Managing Distress & 5.83 & 2.59 & 12 \\
Challenging Behaviour & 7.99 & 3.85 & 9 \\
Other & 14.41 & 2.38 & 8 \\
Non-Cognitive Symptoms & 10.03 & 3.49 & 7 \\
Neuropsychiatric Symptoms & 9.96 & 3.57 & 3 \\
Agitation & 9.07 & 2.81 & 2 \\
Dysregulatory behaviours & 10.85 & 2.33 & 1 \\
Unusual behaviours & 9.36 & 2.91 & 0 \\
Socially unacceptable/ inappropriate & 12.09 & 2.48 & 0 \\
behaviour & & & \\
\hline
\end{tabular}

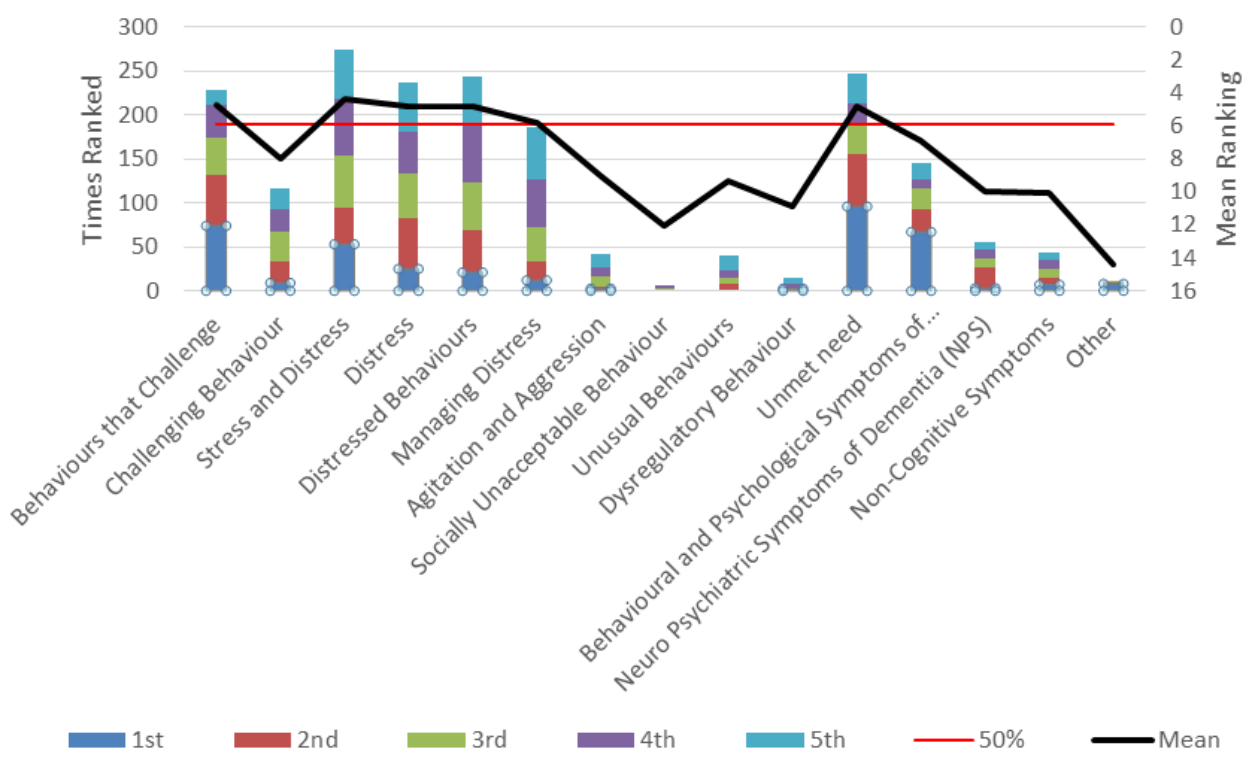

Figure 1 Bar chart demonstrating frequency of a top 5 ranking for each term and the mean ranking I. 


\subsection{Qualitative Results}

Theme relating to usability and breadth of a given term, ethics, causation, hope for change and emotions were extracted from qualitative data analysis in relation to what participants liked and disliked about terminology for this paradigm.

\subsubsection{Theme 1: Generally Aim for User Friendliness and Breadth}

User Friendly. Participants liked terms which they considered to be 'easy to understand', that is 'simple', 'clear and descriptive' - 'it is what it says it is'. Terms were disliked if they were considered 'complicated', 'confusing' or 'meaningless'. However there was no agreement on what was 'overcomplicated jargon' and 'easy to grasp' with examples of likes and dislikes given for the same term. For example: 'This term avoids and euphemism or ambiguity' (referring to BtC); 'I don't like behaviour that challenges it's a meal mouthed alternative to challenging behaviour' (referring to $\mathrm{BtC}$ ).

There was recognition that the terminology was used by a range of people including care staff, health and social care professionals, families and people with dementia so had to be something that could be understood by all, 'it's a simple term and understandable by all' (referring to Distress). To be user friendly, terminology also needs to be 'well known' and 'widely used', however there was considerable variation across settings and professional groups as to what was considered 'established' and 'familiar'.

Participants observed that when terms become widely used, their meaning becomes lost or diluted over time:

'stress and distress is widely used in my area. It originally termed referring to 'stress' in caregivers and 'distress' in the client. This distinction has been lost in everyday use. Staff will speak of clients exhibiting 'stress and distress' (with the word stress being superfluous here). It sounds annoying, and staff will refer to it as jargon-not realising the term is now widely misused' (referring to Stress and Distress).

Broad versus Specific. Some participants preferred terms such as BPSD and 'distressed behaviours' as these were considered to be 'general', 'broad', 'all-encompassing' 'umbrella terms' that could capture a wide range of symptoms and behaviours. However others disliked broad terms since they were not specific enough to be useful and did not convey important information such as severity, or who was being challenged or in what context, "I am aware that distress is being popular but I don't think that it is specific enough' (referring to Distress).

The specific term of aggression and other overt forms of behaviour were considered of limited value in terms of both ameliorative intervention and underlying concepts of 'intent' by the person with dementia 'challenging behaviour is often seen as a behaviour that cannot be managed or people are deliberately behaving in a specific way, it's often linked to being aggressive' (referring to Aggression/Agitation).

\subsubsection{Theme 2 Causation: Linking Behaviour to Dementia and Social Constructs}

Broadly associated with causation, two sub-themes emerged, reflecting the balance of views that conceptualise behaviour changes as rooted solely in the dementia; and those that acknowledge the contribution of the social/interpersonal environment. 
Behaviour and Dementia. There was agreement that some terminology (described as 'medical') explicitly linked behaviour to dementia, implying that is was a direct consequence of dementia (terms such as BPSD, NPS). Some participants viewed these as a preferred option, stating these terms removed any blame from an individual and were therefore less stigmatising: 'attributes behaviours to dementia rather than creating a culture of blame on the person, irrespective of premorbid personality, more likely to engender empathy and understanding' (referring to BPSD); 'normalises as a possible manifestation of disease that needs MDT formulation, and is not blaming of the individual' (referring to NPS).

However, others strongly disliked these terms claiming that viewing the behaviours solely as the result of dementia placed the problem in a person and not the system, 'I think it can reinforce the excuse of "oh it's just dementia", which can be unhelpful if it prevents us from trying to make positive change in the personas care and environment' (referring to BPSD). The notion of 'symptoms' of dementia was seen to distract from 'valid human responses'. Some felt that this approach was reductionist since it promoted only psychiatric responses and did not imply that positive change was possible. There was concern that peoples' experiences could be too easily dismissed and that such language could accelerate discussions about pharmacological intervention; 'I think terms like NPS are more likely to lead to solely medical interventions in responding to distress in dementia. I would opt to scrap these terms in favour of language that leads to a more holistic approach' (referring to NPS).

Behaviour and the Social Environment. Some participants expressed a preference for terms that emphasised that other people, settings and cultures must be considered linked to options for change: 'addressing the connection between the person's behaviour and the people around them' (referring to $\mathrm{BtC}$ ); ' should give a strong indication of potential for external intervention options' (referring to Unmet Need). A common reason for why some participants preferred BtC for example, was that it was seen to recognise that the challenge lies with others and therefore nonblaming of the person with dementia on the one hand, whilst pointing to the agents of change as caregivers, i.e. where change needed to occur. However, others expressed a strong dislike of terms that were construed within social /environmental perspective as these were seen as 'too subjective' and 'too vague' to be clinically useful.

\subsubsection{Theme 3 Not Blaming or Derogatory}

Participants selected terms that they felt were not stigmatising of people with dementia. Some terms were thought to imply that individuals were 'at fault' and to be 'critical', 'judgemental' or 'blaming'. Although there was disagreement about which terms were ethical, terminology which emphasised shared human experiences such as distress or needs were often reported as less stigmatising. To be ethical terminology should also 'invoke compassion' in the responses of other people and terms including the word distress were often regarded as doing this; 'It promotes a compassionate narrative from the start of discussing a person behaviour' (referring to Stress and Distress). A common measure of whether terms were considered ethical was whether participants felt comfortable using the language with people with dementia and their families; 'Much kinder and more explanatory when speaking to family/carers' (referring to Stress and Distress) and 'very unfriendly to family and patients' (referring to BPSD). 
Some terms evoked strong feelings that since they were thought to be simply beyond use and were 'offensive', 'derogatory' and 'demeaning'. Although there was no agreement about which terms these were, most people disliked the term socially unacceptable behaviour for this reason. Some terms were also described as dangerous to use in that they were seen as labelling a person's character or personality and some were described as having almost criminal undertones; 'It implies deliberate deviancy and is associated with very negative stereotypes' (referring to Socially Unacceptable/Inappropriate behaviour). As well as risk to individuals, such terms were felt to perpetuate the stigma surrounding dementia, 'it distances the person with dementia as someone who should not be part of society'; 'them / us scenario' (referring to Socially Unacceptable / Inappropriate behaviour).

\subsubsection{Theme 4 Hopeful: Linking to Rehabilitation}

There was a preference for terminology that was thought to instil hope for change and 'infer resolution is possible'. Participants preferred terms that indicated a reason for a behaviour or an underlying cause and in doing so implied that change was possible with appropriate support; 'it sets a context for exploring what could be causing the distress and looking for ways to reduce it' (referring to Unmet Need). The term unmet needs in particular, was described as giving a 'call to action' or placing responsibility on others to act and also encouraging 'exploration and understanding'. Participants felt that the wrong term had the power to 'limit thinking', 'create a tone of helplessness' and 'prevent positive change'.

\subsubsection{Theme 5 Reflecting Emotions: Linking Behaviour to Communication}

Some participants liked terminology that they felt conveyed the emotions of the person with dementia in recognition that what was being described was typically 'an unpleasant state' and that action needed to be taken. The focus on emotions was seen to be important in switching focus from overt behaviours to 'normal human reactions' that everyone could relate to. It was felt that a focus on emotions would invoke kindness and compassion and motivate people to help; 'instinctively people want to reduce distress or stress in those they care about, so it improves motivation to engage in interventions to understand and reduce the distress (referring to Stress and Distress). Some participants also wanted terms that reflected the highly charged emotions of others with one participant stating the word challenge, 'acknowledges that carers/staff are challenged by the behaviour the person is presenting with' (referring to $\mathrm{BtC}$ ).

\section{Discussion}

While a recent commentary from Australia discussed the topic of language [38], the strength of our study is that, set in the context of England's 2018 Dementia NICE Guidelines and its 2019 quality standards $[39,40]$, we go beyond commentary to capture wide-ranging professional views on language and terminology for this paradigm. Our study demonstrates dissatisfaction with current language for this paradigm amongst multi-disciplinary professionals who support people with dementia in the UK. 


\subsection{Summary}

The present study demonstrates that 14 terms (Figure 1) reflected most of the terminology known to professionals and stakeholders in the UK. Debate from the initial consultation combined with the survey data demonstrated that participants were strongly motivated to avoid placing responsibility for BPSD/CB on people living with a dementia. The quantitative data demonstrated wide variations between professional groupings on their preferred language. Salience of terms related to 'distress' seen within the quantitative data may be due to the current narrative of practitioners and services in the UK, or an attempt by professionals to understand the paradigm in terms of the experience of people with dementia. However, the qualitative data suggests that this change in terminology has been met with mixed responses given that not all behaviour occurs in the context of distress for the person with dementia, or results in distress for people around them. Capturing the notion of distress in people with dementia will remain a challenge for researchers, clinicians and policy makers, as terms like anxiety, depression and agitation do not appear to equate to emotional distress or their internal experiences [43]. Important themes on language that emerged from the qualitative data related to causality, ethics, hope for rehabilitative care and communicative emotional care.

\subsection{Epistemology}

'Causality' incorporated two divergent epistemological positions, where opinions were divided between bio-medical and person-centered social discourses. The former (such as NPS/ BPSD/ Agitation/non-cognitive symptoms) root the phenomena solely in the dementia whilst the latter (such as Unmet Need; BtC/CB) consider reasons unrelated to dementia for why a person may act as they do. However, given that the paradigm covers such a wide spectrum of symptoms (internal states reported by the person themselves) and signs (observable behaviours) it is likely no one perspective, be this medical, social or psychological can hope to adequately capture this range. It is hard, for example, to construct the experience of hallucinations as anything other than biological at its core.

Bio-medical terms were thought to reduce the attribution of blame, but some participants felt that they undermined the search for meaning and understanding. Opinions were divided between the advantages of psychiatric terminology versus the psychological approach. The former acknowledges potential distress in people with dementia, and avoids negative connotations of 'mis' behaviour that undermined dignity; the latter, focusses on understanding 'normal' reactions to frustrating or frightening situations, detecting the sometimes idiosyncratic causes for why people act as they do [17, 18, 44-47]. Lived experiences of 'disturbed behaviour' in people with dementia residing in care homes demonstrate the effects of stigma associated with dementia where an individual can feel 'lost in disorder', 'set aside' and 'trapped' [48].

Definitions of $\mathrm{BtC} / \mathrm{CB}$ were seen by some to advance the case for socially construed approaches that acknowledge the responsibility of others in the interpersonal environment to understand and respond appropriately to psychological needs of the person with dementia. Socially construed considerations associated with caregivers reflect another important paradigm gap [21, 49]. It is often caregiver distress and failure to cope that leads to referral to health professionals [17]; nonpharmacological interventions recommended as first-line approaches are context-dependant, 
usually involving families and staff carers; contextual differences are important for outcome measurement $[19,50]$; and the emotional responses towards an individual affected by dementia are often not only determined by the behaviour, but by carer attributes such as depression in family carers [51, 52]. Stigma associated with dementia can, cause some family carers to experience a deep sense of loss, shame, detachment from the person and fear of an inevitable loss of identity which becomes linked to their reports of BPSD and their challenges in providing support [53]. Organisational factors and staff mood in care homes are also associated with raised levels of reported CB $[54,55]$.

\subsection{Naming the Paradigm}

Unfortunately, carefully constructed definitions for $\mathrm{CB} / \mathrm{BtC}$ that highlight the important contribution of the social environment rarely inform the narrative of this paradigm. This was observed by some participants who noted that the terms $C B$ and $B t C$ have come to be equated with BPSD, and have lost their meaning. Indeed some participants felt that language loses its meaning, and can become diluted or obsolete over time, echoing a comment from an Australian group on the \#banBPSD debate [38]. The question of whether a collective noun is helpful or even possible given the plethora of terms already in use remains. The Australian group recommend using 'the verb "to understand" when talking about a person's behaviour (e.g., "understanding changed behaviours as a result of dementia" pp 1112; line 14-16 [38]. However we note that the notion of 'understanding behaviour in dementia' outlined at the turn of this century with the seminal text on $C B$ [15], does not appear to have resulted in a concept that fully integrates the contribution of the social/interpersonal environment in causation and rehabilitation. The term $\mathrm{CB}$ in dementia was initially drawn from the field of learning disability, to shift the paradigm towards a rehabilitation approach implying that change in behaviour was possible. However within the intellectual disability field, there are now concerns that terms such as CB can result in diagnostic overshadowing where behaviour is attributed to disabilities, instead of practices resulting in failure to examine other causes such as underlying physical health problems or support networks [56].

Given these challenges, a key concern is the potential for nihilism towards refining language for this paradigm. Wendy Mitchell [57], who lives with dementia has pointed to the need for positive language associated with the paradigm ([57]; 0406 16). The debate on diagnostic overshadowing associated with the BPSD/CB paradigm reflects growing recognition, stimulated by people with dementia and their advocates, to approach dementia through a human rights perspective [58, 59]. This may then ensure that disabilities are no longer perceived to be the sole results of defects in people, but also as the consequences of their relationships and environments [60]. A human rights approach has scope to pave the way for neutralising underlying stigma within caregiving environments and preventing nihilism about refined language and terminology for this paradigm.

\subsection{Ethics and Morality}

The qualitative data strongly reinforce the need to review language in this paradigm, on ethical and moral grounds, since language can directly influence the support given to people with dementia and their families. Participants shared concerns that language could diminish personhood and in doing so can put people with dementia at risk of abuse by making others view 
them as less then human, deliberately at fault or beyond help. Similar fears have been expressed by people living with dementia, their families and advocates through movements such as the \#banBPSD (see for example [61]). Some of the concerns are about the relationship of language and harmful responses, particularly with respect to use of pharmaceutical agents (30/09 2018 [62]) and Electroconvulsive Therapy (13/01/2019 [63]). A potential position to begin to our debate on language for this paradigm could be informed by MaCauley's poignant observation that people with dementia 'Respond in Reasonable ways to Adverse Conditions and Circumstances - RRACC [61].

\subsection{Hope: Towards Person-Centred Environments}

Participants most preferred terms were those that they felt instilled hope that change was possible and that promoted curiosity. Many felt that this was achieved through language which positions behavior as shared human reactions or responses. Terms such as 'Unmet Need' that were thought by some to achieve this position, were not without criticism. The importance of language that reflected emotions of people with dementia and those around them was described as a key to instilling empathy, compassion and timely resolution of behavioral challenges (see Chapter 9 [15]) through positive communication. This echoes the call by Mitchell, Dupuis, \& Kontos [64] to create language that inspires and enables respect, love, creativity, and compassionate relating' ( $\mathrm{p} 14$ ) and advocacy movement calls for terminology that reflects a 'progressive and expansive view of the person who lives with a diagnosis of dementia' [65]. Studies and texts on resolution therapy, emotion-orientated communication and empathic curiosity in dementia caregiving $[15,66-68]$ have potential for furthering a refined positive language for this paradigm.

\section{6 Limitations}

The main limitation of this study is the lack of involvement of people with dementia and limited involvement of family carers, since our project was initiated to provide guidance to specialist professionals and providers of dementia care. Recruitment of participants was limited to the UK, since this study was part of an ongoing project to guide practitioners and services across the UK. We are aware of differing terms used beyond our shores including 'behaviors of concern' and 'responsive behaviours', and that similar debates about use of language in this paradigm are occurring internationally [38, 69-71], and through social media [57,61,62]. The use of preference rankings of a relatively large number (i.e. 14) of items is also not without limitations. Many items related to 'distress' in some form and none of these were presented in a randomised order. This is was not a systematic survey since participants were a convenience, volunteer sample with mixed representation by differing professionals groups with underrepresentation of some key practitioners, notably 'hands on' care staff working in residential and home care settings. Participants frequently commented on the importance of shared ownership of language with care workers. The issue of response bias in relation to receipt of an invitation to complete the survey must also be acknowledged. Because of the cascading method, it is not possible to say how many individuals received the survey, nor the response rate. It is likely that an individual responding to such a survey invitation is likely to have strong feelings around the subject, regardless of their 
professional background. Given the nature of cascading through a group who were primarily clinical psychologists, findings may be biased towards a psychological person-centred frame.

\section{Conclusions and Next Steps}

Our study was modest but showed a high level of disagreement and dissatisfaction with the terms that are used to describe the most challenging and pressing issues that confront those living with dementia and those caring for them. What is without doubt is that the responsibility to communicate with people living with dementia and their families in language they can understand and relate to is incumbent on the professional, regardless of their discipline. This resonates with the dissatisfaction being expressed by dementia advocacy groups. Hearing these voices is essential in moving this discussion forward, given our finding that participants sought a shared language that is owned and understood by all. The choice of words we use can be perceived as a reflection of our thoughts and feelings. They can show respect or disrespect, and empower or disempower individuals or whole groups of people living with a dementia. Systematic study of the views of people with dementia and their advocates on an alternative language and application of this in research and practice is an important next step. Some authors suggest that the term BPSD might be of help to carers who struggle to make sense of changes in a relative with dementia [38], but this did not accord with the data taken from the small number of family carers who responded to our survey. Systematic study of the family caregiver perspective would complement the findings of our study, although it should be considered that some family carers might use more pejorative terms to describe changes in behaviour that they struggle to understand. A final modified Delphi round of discussion with an expert reference group will examine the findings reported here, to consider what terminology for this paradigm might be relevant to the range of practice contexts, and what related tools might guide practitioners in their routine work with people with dementia and their supporters in the UK. However, future studies of this type will require careful thought about choice of experts, practitioners, family carers, international advocates and people with dementia if trustworthy consensus on language, rehabilitation and associated outcome measurement for this paradigm is to be achieved.

\section{Author Contributions}

All authors were involved in organising and running the stakeholder event and in designing the subsequent survey. EW and HB conducted the qualitative data analysis. HB, EMC, IJ and FD were involved with the quantitative data analysis. EW and EMC prepared the manuscript all authors were involved in reviewing and commenting on drafts.

\section{Funding}

This study is part of a wider programme of work to produce a set of good practice guidelines on how to deliver interventions for Behaviours that Challenge /BPSD, funded by the British Psychological Society, UK.

\section{Competing Interests}

The authors have declared that no competing interests exist. 


\section{References}

1. Fairburn CG, Hope RA. Changes in behaviour in dementia: A neglected research area. $\mathrm{Br} J$ Psychiatry. 1988; 152: 406-407.

2. Hope RA, Fairburn CG. The nature of wandering in dementia: A community-based study. Int J Geriatr Psychiatry. 1990; 5: 239-245.

3. Finkel S, Costa E, Silva J, Cohen G, Miller S, Sartorius N. Behavioral and psychological signs and symptoms of dementia: A consensus statement on current knowledge and implications for research and treatment. Int Psychogeriatr. 1996; 8: 497-500.

4. Finkel S, Silva JC, Cohen G, Miller S, Sartorius N. Behavioral and psychological signs and symptoms of dementia: A consensus statement on current knowledge and implications for research and treatment. Int J Geriatr Psychiatry. 1997; 12: 1060-1061.

5. Lyketsos CG, Carrillo MC, Ryan JM, Khachaturian AS, Trzepacz P, Amatniek J, et al. Neuropsychiatric symptoms in Alzheimer's disease. Alzheimers Dement. 2011; 7: 532-539.

6. Leibing A. "From the periphery to the center: Treating noncognitive, especially behavioral and psychological, symptoms of dementia," in treating dementia: Do we have a pill for that? Ballenger J, Whitehouse P, Lyketsos C, Rabins P, Karlawish J, editors. Baltimore: The Johns Hopkins University Press; 2009.

7. Mendes MF, Cummings JL. Pharmacotherapy of alzheimers disease and other dementias. Dementia a clinical approach (third edition). USA Philadelphia Butterworth Heinmann imprint of Elsvier Science; 2003. p. 559 -582.

8. Chen P, Guarino PD, Dysken MW, Pallaki M, Asthana S, Llorente MD, et al. Neuropsychiatric symptoms and caregiver burden in individuals with Alzheimer's disease: The team-ad va cooperative study. J Geriatr Psychiatry Neurol. 2018; 31: 177-185.

9. Banerjee S. The use of antipsychotic medication for people with dementia: Time for action. London: DoH; 2009.

10. Brechin D, Murphy G, James IA, Codner J. Alternatives to antipsychotic medication: Psychological approaches in managing psychological and behavioural distress in people with dementia2013 19/08/2019. Available from: http://www.psige.org/public/files/BPS\%20FPoP\%20-\%20Alternatives\%20to\%20AntiPsychotic\%20Medication\%20-\%20report\%20-\%20March\%202013.pdf.

11. National Institute for Health and Care Excellence (NICE). Dementia: Assessment, management and support for people living with dementia and their carers nice guideline [ng97] - managing non-cognitive symptoms2018 29/08/2019. Available from: https://www.nice.org.uk/guidance/ng97.

12. Kitwood T, Benson S. The new culture of dementia care. Kitwood T, Benson S, editors. London: Hawker Publications; 1995.

13. Kitwood T. Dementia reconsidered: The person comes first (rethinking ageing). Open University Press; 1997.

14. Kitwood T. Dementia reconsidered, revisited: The person still comes first. Brooker D, editor. London: Open University Press; 2019.

15. Stokes G. Challenging behaviour in dementia: A person centered approach. Winslow Press; 2000.

16. Andrews GJ. Managing challenging behaviour in dementia. BMJ. 2006; 332: 741. 
17. Bird M, Moniz-Cook ED. Challenging behaviour in dementia; a psychosocial approach to intervention in handbook of the clinical psychology of ageing. Woods B, Clare L, editors: Wiley; 2008.

18. Bird M, Llewellyn-Jones RH, Korten A. An evaluation of the effectiveness of a case-specific approach to challenging behaviour associated with dementia. Aging Ment Health. 2009; 13: 73-83.

19. Moniz Cook ED, Swift K, James I, Malouf R, De Vugt M, Verhey F. Functional analysis-based interventions for challenging behaviour in dementia. Cochrane Database Syst Rev. 2012: CD006929.

20. Holle $D$, Halek M, Holle B, Pinkert C. Individualized formulation-led interventions for analyzing and managing challenging behavior of people with dementia - an integrative review. Aging Ment Health. 2016; 13: 73-83.

21. Moniz-Cook E, Hart C, Woods B, Whitaker C, James I, Russell I, et al. Challenge demcare: Management of challenging behaviour in dementia at home and in care homes: Development, evaluation and implementation of an online individualised intervention for care homes; and a cohort study of specialist community mental health care for families. Programme Grants for Applied Research. 2017; 5.

22. National Institute for Health and Clinical Excellence/Social Care Institute for Excellence (NICE/SCIE). Dementia: Supporting people with dementia and their carers in health and social care. Nice clinical guideline ng42.2007 19/08/2019. Available from: https://www.scie.org.uk/publications/misc/dementia/dementia-fullguideline.pdf.

23. James IA. Understanding behaviour in dementia that challenges - a guide to assessment and treatment. London and Philadelphia, PA: Jessica Kingsley; 2011.

24. Evidence briefing: Behaviour that challenges in dementia [Internet]. British Psychological Society. 2018 [cited 16/08/2019]. Available from: https://www.bps.org.uk/news-andpolicy/psychological-dimensions-dementia.

25. National Institute for Health and Care Excellence (NICE). Dementia - assessment, management and support for people living with dementia and their carers. Nice clinical guideline [ng97]. Section 1.7. Managing non-cognitive symptoms [Internet]. 2018 29/08/2019. Available from:

https://www.nice.org.uk/guidance/ng97/chapter/Recommendations\#managing-noncognitive-symptoms.

26. Dementia Behaviour Management Advisory Services. Reboc reducing behaviours of concern2012. Available from: https://dementia.com.au/resources/library/understandingand-responding-to-behaviour-resources/reboc-guide-reducing-behaviours-of-concern.html.

27. Laver K, Cumming RG, Dyer SM, Agar MR, Anstey KJ, Beattie E, et al. Clinical practice guidelines for dementia in australia. The Medical journal of Australia [Internet]. 2016 02/09/2019; 204(5):[191-193 pp.]. Available from: https://sydney.edu.au/medicine/cdpc/documents/resources/LAVER_Dementia_Guidleines_r ecommendations_PRVW\%20(4).pdf.

28. Ballard CG, O'Brien J, James I, Swann A. Dementia: Management of behavioural and psychological symptoms. Oxford: Oxford University Press; 2001. 
29. Vickland V, Chilko N, Draper B, Low LF, O'Connor D, Brodaty H. Individualized guidelines for the management of aggression in dementia - part 2: Appraisal of current guidelines. Int Psychogeriatr. 2012; 24: 1125-1132.

30. Moniz-Cook E, Stokes G, Agar S. Difficult behaviour and dementia in nursing homes: Five cases of psychosocial intervention. Clin Psychol Psychother. 2003; 10: 197-208.

31. Salzman C, Jeste DV, Meyer RE, Cohen-Mansfield J, Cummings J, Grossberg GT, et al. Elderly patients with dementia-related symptoms of severe agitation and aggression: Consensus statement on treatment options, clinical trials methodology, and policy. J Clin Psychiatry. 2008; 69: 889-898.

32. Soto M, Abushakra S, Cummings J, Siffert J, Robert P, Vellas B, et al. Progress in treatment development for neuropsychiatric symptoms in Alzheimer's disease: Focus on agitation and aggression. A report from the eu/us/ctad task force. J Prev Alzheimers Dis. 2015; 2: 184-188.

33. Soto M, Andrieu S, Nourhashemi F, Ousset PJ, Ballard C, Robert P, et al. Medication development for agitation and aggression in Alzheimer disease: Review and discussion of recent randomized clinical trial design. Int Psychogeriatr. 2015; 27: 181-197.

34. Volicer L, Vitturi B. Neurobiological differences between aggression and agitation in persons with dementiaaffiliation. J Neurol Res Ther. 2019; 2: 28-36.

35. Cummings J, Mintzer J, Brodaty H, Sano M, Banerjee S, Devanand DP, et al. Agitation in cognitive disorders: International psychogeriatric association provisional consensus clinical and research definition. Int Psychogeriatr. 2015; 27: 7-17.

36. Sano $M$, Soto $M$, Carrillo $M$, Cummings J, Hendrix $S$, Mintzer J, et al. Identifying better outcome measures to improve treatment of agitation in dementia: A report from the eu/us/ctad task force. J Prev Alzheimers Dis. 2018; 5: 98-102.

37. Scottish Government. Promoting excellence: A framework for all health and social services staff working with people with dementia and their family and carers2011 19/08/2019. Available from: https://www.nes.scot.nhs.uk/media/1523859/promoting_excellence.pdf.

38. Cunningham C, Macfarlane S, Brodaty $H$. Language paradigms when behaviour changes with dementia: \#banbpsd. Int J Geriatr Psychiatry. 2019; 34: 1109-1113.

39. National Institute for Health and Care Excellence (NICE). Dementia - quality standard [qs184]2019 19/08/2019. Available from:

https://www.nice.org.uk/guidance/qs184/resources/dementia-pdf-75545721373381.

40. National Institute for Health and Care Excellence (NICE). Dementia - assessment, management and support for people living with dementia and their carers. Nice clinical guideline [ng97]2018 19/08/2019. Available from: https://www.nice.org.uk/guidance/ng97.

41. Joffe $\mathrm{H}$. Thematic analysis. Qualitative research methods in mental health and psychotherapy 1.2012.

42. Braun V, Clarke V. Using thematic analysis in psychology. Q Res Psychol. 2006; 3: 77-101.

43. Petty S, Dening T, Coleston DM, Griffiths A. Dementia: Beyond disorders of mood. Aging Ment Health. 2019; 23: 525-528.

44. Bird $\mathrm{M}$, Jones RH, Korten A, Smithers H. A controlled trial of a predominantly psychosocial approach to bpsd: Treating causality. Int Psychogeriatr. 2007; 19: 874-891.

45. Stokes G. And still the music plays. London: Hawker Press; 2008.

46. Duffy F. 'Look at all of me'- a clear model for dementia care. J Dement Care. 2016; 24: 27-30. 
47. Duffy F. Clear dementia care. A model to assess and and address unmet need. Jessica Kingsley Publishers; 2019.

48. Graneheim UH, Jansson L. The meaning of living with dementia and disturbing behaviour as narrated by three persons admitted to a residential home. J Clin Nurs. 2006; 15: 1397-1403.

49. Moniz-Cook E. Psychosocial approaches to behaviours that challenge care. In evidence-based practice in dementia for nurses and nursing students. Harrison Dening K, editor. London: Jessica Kingsley; 2019.

50. Gitlin LN, Marx KA, Stanley IH, Hansen BR, Van Haitsma KS. Assessing neuropsychiatric symptoms in people with dementia: A systematic review of measures. Int Psychogeriatr. 2014; 26: 1805-1848.

51. de Vugt ME, Stevens F, Aalten P, Lousberg R, Jaspers N, Winkens I, et al. Do caregiver management strategies influence patient behaviour in dementia? Int J Geriatr Psychiatry. 2004; 19: 85-92.

52. Hinchliffe AC, Hyman IL, Blizard B, Livingston G. Behavioural complications of dementia? Can they be treated? Int J Geriatr Psychiatry. 1995; 10: 839-847.

53. Feast A, Orrell M, Charlesworth G, Melunsky N, Poland F, Moniz-Cook E. Behavioural and psychological symptoms in dementia and the challenges for family carers: Systematic review. Br J Psychiatry. 2016; 208: 429-434.

54. Baillon S, Scothern G, Neville PG, Boyle A. Factors that contribute to stress in care staff in residential homes for the elderly. Int J Geriatr Psychiatry. 1996; 11: 219-226.

55. Moniz-Cook E, Woods R, Gardiner E. Staff factors associated with perception of behaviour as 'challenging' in residential and nursing homes. Aging Ment Health. 2000; 4: 48-55.

56. Chan J, Arnold S, Webber L, Riches V, Parmenter T, Stancliffe R. Is it time to drop the term 'challenging behaviour'? Learning Disability Practice. 2012; 15: 36-38.

57. Mitchell W. Stay positive: Use of language in dementia research2016 29/08/2019. Available from:

https://www.dc.nihr.ac.uk/blog/stay-positive-use-of-language-in-dementiaresearch/3534.

58. Swaffer K. Afterword. In: Kitwood, Brooker, editors. Dementia reconsidered, revisited: The person still comes first. London: Open University Press; 2019. p. 178-180

59. Swaffer K. Human rights disability and dementia. Aust J Dement Care [Internet]. 2018 05/09/2019. Available from: https://journalofdementiacare.com/human-rights-disability-anddementia/.

60. World Health Organization. International classification of functioning, disability and health2012 19/08/2019. Available from: http://www.who.int/classifications/icf/en.

61. MaCaulay S. Take off the blindfolds and \#banbpsd: An open letter to the worldwide dementia community 2018 Available from: http://myalzheimersstory.com/2018/06/09/take-off-the-blindfolds-and-banbpsd-an-openletter-to-the-worldwide-dementia-community/.

62. Swaffer K. Rethinking dementia banbpsd2018 29/08/2019. Available from: https://kateswaffer.com/2018/09/30/rethinking-dementia-banbpsd/.

63. Swaffer K. We need to ban bpsd before it's too late...2019 29/08/2019. Available from: https://kateswaffer.com/2019/01/13/we-need-to-ban-bpsd-before-its-too-late/.

64. Mitchell GJ, Dupuis SL, Kontos PC. Dementia discourse: From imposed suffering to knowing other-wise. Journal of Applied Hermeneutics. 2013. 
65. Barsness S, Bisiani L, Greenwood D, Macaulay S, Power A, Swaffer K. Rethinking dementia care. Aust J Dement Care [Internet]. 2018 03/09/2019; 7: 8. Available from: https://kateswaffer.files.wordpress.com/2018/08/screen-shot-2018-08-11-at-8-02-12am.png.

66. Finnema $E$, Dröes RM, Ribble $M$, Van Tillberg $W$. The effects of emotion-orientated approaches in the care of persons suffering from dementia: A review of the literature. Int J Geriatr Psychiatry. 2000; 15: 141-161.

67. Rewston C, Moniz-Cook E. Understanding and alleviating emotional distress. In: Downs MG, Bowers BJ, editors. Excellence in dementia care: Principles and practice. Berkshire: Open University Press McGraw Hill; 2008. p. 249-263.

68. McEvoy P, Plant R. Dementia care: Using empathic curiosity to establish the common ground that is necessary for meaningful communication. J Psychiatr Ment Health Nurs. 2014; 21: 477482.

69. Alzheimer's Australia. Dementia friendly language: Position paper 42009 19/08/2019. Available from:

https://www.dementia.org.au/sites/default/files/20090600_Nat_NP_4DemFriendLang.pdf.

70. Alzheimer's Society Canada. Person centred language guidelines2017 19/08/2019. Available from:

https://alzheimer.ca/sites/default/files/2017-11/Person_Centred_Language_Guidelines-e.pdf.

71. Alzheimer's Society of Ireland. Dementia friendly language2018 19/08/2019. Available from: https://alzheimer.ie/wp-content/uploads/2018/11/Dementia-Friendly-Language.pdf.

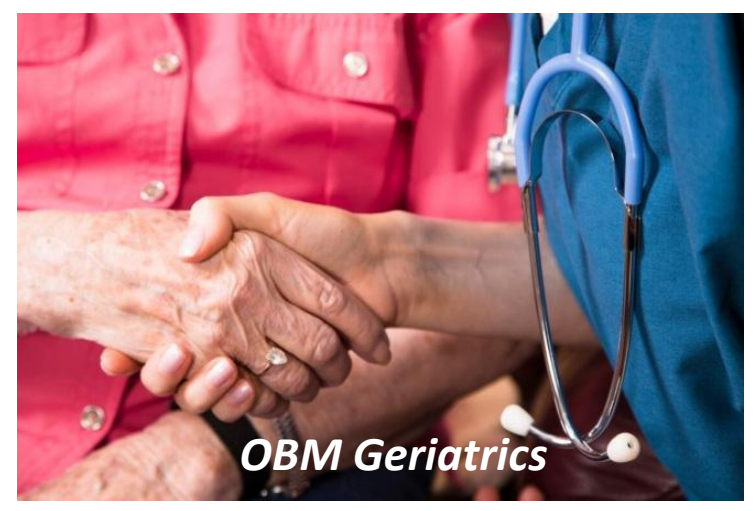

Enjoy $O B M$ Geriatrics by:

1. Submitting a manuscript

2. Joining in volunteer reviewer bank

3. Joining Editorial Board

4. Guest editing a special issue

For more details, please visit: http://www.lidsen.com/journals/geriatrics 